LAWRENCE LIVERMORE NATIONAL LABORATORY

\title{
Effective Determination of Coexistence Curves using Reversible-Scaling Molecular Dynamics Simulations
}

M. de Koning, A. Antonelli, S. Yip

December 18, 2000

Materials Research Society Fall 2000 Meeting, Boston, MA

November 27-December 1, 2000 


\section{DISCLAIMER}

This document was prepared as an account of work sponsored by an agency of the United States Government. Neither the United States Government nor the University of California nor any of their employees, makes any warranty, express or implied, or assumes any legal liability or responsibility for the accuracy, completeness, or usefulness of any information, apparatus, product, or process disclosed, or represents that its use would not infringe privately owned rights. Reference herein to any specific commercial product, process, or service by trade name, trademark, manufacturer, or otherwise, does not necessarily constitute or imply its endorsement, recommendation, or favoring by the United States Government or the University of California. The views and opinions of authors expressed herein do not necessarily state or reflect those of the United States Government or the University of California, and shall not be used for advertising or product endorsement purposes.

This is a preprint of a paper intended for publication in a journal or proceedings. Since changes may be made before publication, this preprint is made available with the understanding that it will not be cited or reproduced without the permission of the author.

This work was performed under the auspices of the United States Department of Energy by the University of California, Lawrence Livermore National Laboratory under contract No. W-7405-Eng-48.

This report has been reproduced directly from the best available copy.

Available electronically at http://www.doc.gov/bridge

Available for a processing fee to U.S. Department of Energy

And its contractors in paper from

U.S. Department of Energy

Office of Scientific and Technical Information

P.O. Box 62

Oak Ridge, TN 37831-0062

Telephone: (865) 576-8401

Facsimile: (865) 576-5728

E-mail: reports@adonis.osti.gov

Available for the sale to the public from

U.S. Department of Commerce

National Technical Information Service

5285 Port Royal Road

Springfield, VA 22161

Telephone: (800) 553-6847

Facsimile: (703) 605-6900

E-mail: orders@ntis.fedworld.gov

Online ordering: http://www.ntis.gov/ordering.htm

OR

Lawrence Livermore National Laboratory

Technical Information Department's Digital Library

http://www.llnl.gov/tid/Library.html 


\title{
Effective Determination of Coexistence Curves using Reversible-Scaling Molecular Dynamics Simulations
}

\author{
Maurice de Koning ${ }^{1,2}$, Alex Antonelli ${ }^{3}$, and Sidney Yip ${ }^{1}$ \\ ${ }^{1}$ Department of Nuclear Engineering, Massachusetts Institute of Technology, Cambridge, MA \\ 02139-4307, U.S.A. \\ ${ }^{2}$ Lawrence Livermore National Laboratory, University of California, Livermore, CA 94550- \\ 9234, U.S.A. \\ ${ }^{3}$ Instituto de Física Gleb Wataghin, Universidade Estadual de Campinas, Unicamp 13083-970, \\ Campinas, São Paulo, Brazil
}

\begin{abstract}
We present a simulation technique that allows the calculation of a phase coexistence curve from a single nonequilibrium molecular dynamics (MD) simulation. The approach is based on the simultaneous simulation of two coexisting phases, each in its own computational cell, and the integration of the relevant Clausius-Clapeyron equation starting from a known coexistence point. As an illustration of the effectiveness of our approach we apply the method to explore the melting curve in the Lennard-Jones phase diagram.
\end{abstract}

\section{INTRODUCTION}

One of the most challenging applications of computer simulation techniques concerns the determination of the phase behavior of a substance of interest. Given that the size of the systems studied in typical simulations is relatively small, a direct observation of phase coexistence in a computational cell is problematic due to the interface dividing the coexisting phases. In order to avoid such problems one usually adopts an indirect route in which the coexistence conditions are determined by considering the thermodynamic properties of each phase individually. An effective methodology for the computation of phase equilibria using such an approach is the Clausius-Clapeyron integration (CCI) method introduced by Kofke [1-5], in which the relevant Clausius-Clapeyron differential equation is solved numerically. For coexistence in the pressure/temperature domain, for example, this equation has the well-known form

$$
\frac{d P}{d T}=\frac{\Delta H}{T \Delta V},
$$

where $\Delta H$ and $\Delta V$ are, respectively, the molar enthalpy and volume differences between two coexisting phases $A$ and $B$. In Kofke's implementation this equation is discretized and integrated numerically by conducting a series of equilibrium simulations along which the temperature (or pressure) varies on a discrete mesh. Each simulation considers both phases simultaneously, in separate computational cells, and measures the differences $\Delta H$ and $\Delta V$ corresponding to the current values of temperature and pressure. Given an initial coexistence condition, the rest of the coexistence curve is then mapped out by updating the coexistence pressure (or temperature) according to Eq. (1) using a predictor-corrector integrator. In this fashion each equilibrium simulation provides one new point on the coexistence curve. 
In this paper we present an alternative implementation of the CCI concept which enables the determination of an entire coexistence curve from only one nonequilibrium simulation. The approach is based on the Reversible-Scaling free-energy method [6-8] and replaces the integration along a discrete series of equilibrium states by one along a quasi-continuous sequence of nonequilibrium states. We illustrate the effectiveness of our approach by exploring the melting curve in the Lennard-Jones phase diagram.

\section{METHODOLOGY}

\section{Reversible Scaling}

The Reversible-Scaling (RS) technique [6-8] is based on an equivalence relation between the partition functions of a given system of interest and its associated scaled system, whose potential energy function is constructed by scaling the original one by a positive factor $\lambda$ :

$$
U_{\text {scaled }}\left(\left\{\vec{r}_{i}\right\} ; \lambda\right) \equiv \lambda U_{\text {system }}\left(\left\{\vec{r}_{i}\right\}\right)
$$

where the $\vec{r}_{i}$ denote the $N$ particle positions. Considering the isobaric-isothermal partition functions [9] for both systems one finds that their configurational Gibbs free energies are related according to

$$
G_{\text {system }}\left(N, P_{0}, T\right)=\frac{T}{T_{0}} G_{\text {scaled }}\left(N, \lambda P_{0}, T_{0} ; \lambda\right),
$$

where $P_{0}$ and $T$ are the pressure and temperature in the system of interest, and $P_{\text {scaled }}=\lambda P_{0}$ and $T_{0}=\lambda T$ are the corresponding values in the scaled system. This relation implies that the problem of computing the free energy of the system of interest as a function of temperature $T$ is completely equivalent to the problem of evaluating the free energy of the scaled system as a function of the scaling parameter $\lambda$ at a constant temperature $T_{0}$.

The latter can be addressed very effectively within the framework of the coupling parameter formalism of thermodynamic integration (TI) [9], according to which the free energy of the scaled system may be written as

$$
G_{\text {scaled }}\left(N, \lambda P_{0}, T_{0} ; \lambda\right)=G_{\text {scaled }}\left(N, \lambda_{\text {ref }} P_{0}, T_{0} ; \lambda_{\text {ref }}\right)+W_{\text {rev }}(\lambda)
$$

where $G_{\text {scaled }}\left(N, \lambda_{\text {ref }} P_{0}, T_{0} ; \lambda_{\text {ref }}\right) \equiv G_{\text {system }}\left(N, P_{0}, T_{0} / \lambda_{\text {ref }}\right)$ is a reference free-energy value assumed to be known beforehand, and where

$$
W_{\text {rev }}(\lambda) \equiv \int_{\lambda_{\text {ref }}}^{\lambda} d \lambda^{\prime}\left(\left.\frac{\partial G_{\text {scaled }}(\lambda)}{\partial \lambda}\right|_{\lambda^{\prime}}\right)=\int_{\lambda_{\text {ref }}}^{\lambda} d \lambda^{\prime}\left[P_{0}\langle V\rangle_{\lambda^{\prime}}+\left\langle U_{\text {system }}\right\rangle_{\lambda^{\prime}}\right]
$$


is the cumulative reversible work function associated with the scaling coordinate $\lambda$. Here, $\langle V\rangle$ and $\left\langle U_{\text {system }}\right\rangle$ denote ensemble averages of, respectively, the volume and potential energy over the $\left(N, \lambda P_{0}, T_{0} ; \lambda\right)$ ensemble for the scaled system.

The function $W_{r e v}(\lambda)$ can be estimated very efficiently using the adiabatic switching (AS) nonequilibrium method [8], in which the scaling parameter $\lambda=\lambda(t)$ is a function of time that varies continuously between initial and final values $\lambda(0)=\lambda_{\text {ref }}$ and $\lambda\left(t_{s}\right)=\lambda_{f}, t_{s}$ being the switching time of the AS simulation. In this manner one obtains a dynamical estimator for the function $W_{r e v}(\lambda)$ from a single AS simulation of duration $t_{s}$ :

$$
W_{d y n}[\lambda(t)]=\int_{0}^{t} d t^{\prime} \dot{\lambda}\left(t^{\prime}\right)\left[P_{0} V\left(t^{\prime}\right)+U_{\text {system }}\left(t^{\prime}\right)\right], \quad 0 \leq t \leq t_{s},
$$

where the ensemble averages of the volume and potential energy in Eq. (5) have been replaced by their instantaneous values during the AS simulation. Evidently, $W_{d y n}[\lambda(t)]$ is a biased estimator for $W_{\text {rev }}(\lambda)$ since the AS process is irreversible in nature and subject to dissipative entropy production. Accordingly, the dynamical estimator (8) will be rigorously equal to $W_{\text {rev }}(\lambda)$ only in the limit $t_{s} \rightarrow \infty$ when the process becomes ideally adiabatic. In practice, however, the convergence of $W_{d y n}[\lambda(t)]$ with increasing $t_{s}$ is remarkably rapid, allowing an accurate determination of $W_{r e v}(\lambda)$ and $G_{\text {system }}\left(N, P_{0}, T\right)$ (through Eqs. (4) and (3)), from a single and relatively short RS-AS simulation. Moreover, any small systematic errors caused by finite switching times can be eliminated using a simple hysteresis analysis procedure [10,11].

\section{Clausius-Clapeyron Reversible Scaling}

The RS approach can be extended in a manner that allows efficient and accurate integration of the Clausius-Clapeyron equation for two coexisting phases. In analogy to Eq. (4), the free energies of the scaled systems associated with the phases $A$ and $B$ can be written as

$$
G_{\text {scaled }}^{A, B}\left(N, \lambda P_{0}(\lambda), T_{0} ; \lambda\right)=G_{\text {scaled }}^{A, B}\left(N, \lambda_{\text {ref }} P_{0}\left(\lambda_{\text {ref }}\right), T_{0} ; \lambda_{\text {ref }}\right)+W_{\text {rev }}^{A, B}(\lambda)
$$

where the pressure $P_{0}$ in the systems of interest now varies explicitly as a function of temperature (and thus as a function of $\lambda$ in the corresponding scaled systems). The corresponding cumulative reversible work functions for both phases are given by

$$
W_{\text {rev }}^{A, B}(\lambda)=\int_{\lambda_{\text {ref }}}^{\lambda} d \lambda^{\prime}\left[P_{0}\left(\lambda^{\prime}\right)+\left.\lambda^{\prime} \frac{d P_{0}}{d \lambda}\right|_{\lambda^{\prime}}\right]\langle V\rangle_{\lambda^{\prime}}^{A, B}+\int_{\lambda_{\text {ref }}}^{\lambda} d \lambda^{\prime}\left\langle U_{\text {system }}\right\rangle_{\lambda^{\prime}}^{A, B} .
$$

Now, suppose the phases $A$ and $B$ are in coexistence at some temperature $T_{\text {ref }}=T_{0} / \lambda_{\text {ref }}$ and pressure $P_{\text {ref }}$. In order to maintain the phases in coexistence upon an infinitesimal change $d \lambda$ in 
the scaled systems, the associated reversible work increments for phases $A$ and $B$ must be equal. In order to satisfy this condition, the derivative $d P_{0} / d \lambda$ must obey

$$
\lambda \frac{d P_{0}}{d \lambda}=-P_{0}(\lambda)-\frac{\left\langle U_{\text {system }}\right\rangle_{\lambda}^{A}-\left\langle U_{\text {system }}\right\rangle_{\lambda}^{B}}{\langle V\rangle_{\lambda}^{A}-\langle V\rangle_{\lambda}^{B}}
$$

which is simply the Clausius-Clapeyron equation within the RS formulation. Implementing Eq. (9) within the AS scheme then leads to a dynamical "equation of motion" for the evolution of the coexistence pressure:

$$
\frac{d P_{0}[\lambda(t)]}{d t}=-\frac{\dot{\lambda}(t)}{\lambda(t)}\left(P_{0}[\lambda(t)]+\frac{U_{\text {system }}^{A}(t)-U_{\text {system }}^{B}(t)}{V^{A}(t)-V^{B}(t)}\right)
$$

Given an initial coexistence condition, the integration of this equation then provides a dynamical estimator for an entire coexistence curve from a single RS-AS simulation in which both phases are considered simultaneously.

\section{APPLICATION: MELTING CURVE IN LENNARD-JONES SYSTEM}

As an illustration of the effectiveness of the Clausius-Clapeyron RS-AS technique, we computed the fcc solid-liquid coexistence boundary in the Lennard-Jones (LJ) system. For both phases we employed cubic computational cells containing 500 particles subject to standard periodic boundary conditions. The cut-off radius was selected at $r_{c}=3.5 \sigma$ and standard longrange corrections were applied to the measured energies and pressures [9]. The MD simulations were carried out using isobaric-isothermal dynamics based on a combination of the Nosé-Hoover thermostat and Andersen barostat. The corresponding equations of motion were integrated using the leapfrog algorithm with a time step $\Delta t=0.002$ (LJ units).

First, we determined an initial point on the melting line by computing the Gibbs free energies of the LJ solid and liquid as a function of temperature at a pressure $P_{0}=1 \varepsilon / \sigma^{3}$ using the RS-AS technique. The functional form of $\lambda(t)$ was chosen to be linear in $t$. Figure 1 shows the converged results for the configurational Gibbs free energies per particle as a function of temperature, obtained using switching times of $t_{s}=10\left(5 \cdot 10^{3} \mathrm{MD}\right.$ steps $)$ and $t_{s}=50$ $\left(2.5 \cdot 10^{4}\right.$ steps) LJ time units for the solid and liquid phases, respectively. As a check we conducted independent free-energy computations at the upper and lower limits of the temperature intervals covered by the RS-AS simulations. The results of these calculations, shown as the symbols in the plot, indicate an excellent agreement with the RS-AS data. From the crossing of both free-energy curves the coexistence condition for the pressure $P_{0}=1 \varepsilon / \sigma^{3}$ was found at a temperature $T_{m}=(0.777 \pm 0.001) \varepsilon / k_{B}$. 


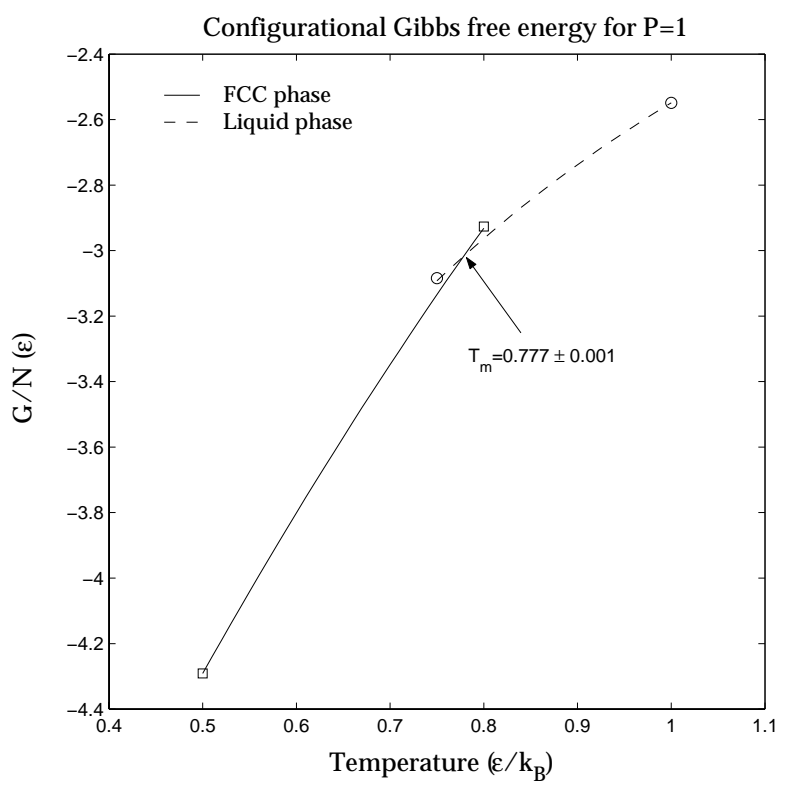

Figure 1. Configurational Gibbs free energy as a function of temperature for the Lennard-Jones FCC and liquid phases.

Starting from this initial coexistence point we applied the Clausius-Clapeyron RS-AS technique to extrapolate the melting line to the temperature $T=7.777$. Figure 2 shows the results obtained for three values of the switching time: $t_{s}=5$ ( full curve, $1 \cdot 10^{3} \mathrm{MD}$ steps), $t_{s}=20$ (dotted curve, $1 \cdot 10^{4} \mathrm{MD}$ steps), and $t_{s}=200$ ( dashed curve, $1 \cdot 10^{5} \mathrm{MD}$ steps). For comparison, the equilibrium CCI results from [4] (obtained for 500 and 432 particles in the solid and liquid phases, respectively) are shown as the square symbols.

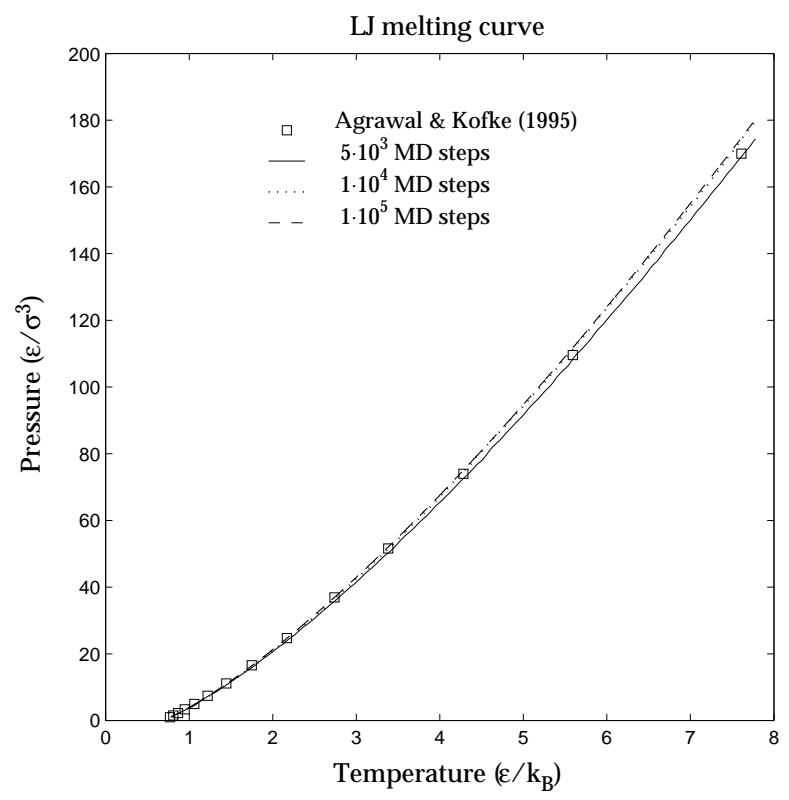

Figure 2. Clausius-Clapeyron RS-AS melting curves for the LJ system. Symbols represent data from [4]. 
The agreement between the Clausius-Clapeyron RS-AS results and those obtained in [4] is excellent for all temperatures below $T=4.0$. For higher temperatures our results predict a slightly higher coexistence pressure, reaching a discrepancy of $2.5 \%$ at $T=7.61$. This discrepancy is possibly related to the fact that our calculations were carried out using a fixed cutoff radius for the potential, while the results in [4] were based on a variable cut-off radius. Considering the computational overhead associated with both methods, the Clausius-Clapeyron RS-AS technique is found to be considerably more efficient than the equilibrium method used in [4]. Given that each data point in the calculations of [4] required at least $4.2 \cdot 10^{4}$ Monte Carlo (MC) trials, the nonequilibrium implementation of CCI is about a factor 15 less expensive, showing a converged coexistence boundary using only $1 \cdot 10^{4} \mathrm{MD}$ steps to cover the entire temperature range between $T=0.777$ and $T=7.777$.

\section{SUMMARY}

We presented a new implementation of the Clausius-Clapeyron integration procedure for the determination of phase coexistence boundaries using atomistic simulation techniques. Our approach is based on the Reversible-Scaling free energy method and allows the calculation of an entire coexistence curve from a single, relatively short nonequilibrium simulation. Application of the technique to the calculation of the melting curve in the Lennard-Jones phase diagram shows that the present nonequilibrium method is significantly less expensive than the original equilibrium implementation. Given the demonstrated efficiency, the method appears to be useful for the calculation of first-principles coexistence curves.

\section{ACKNOWLEDGMENTS}

M.K. acknowledges support from the Brazilian agency FAPESP under Contract No. 97/14290-6, and from the U.S. Department of Energy through the University of California Lawrence Livermore National Laboratory under contract No. W-7405-Eng-48. A.A. gratefully acknowledges support from FAPESP, CAPES and CNPq, all of them Brazilian funding agencies. S.Y acknowledges support by Lawrence Livermore National Laboratory (ASCI-Level 2 grant), AFOSR (F49620-00-10082) and NSF (DMR-9980015).

\section{REFERENCES}

1. D.A. Kofke, J. Chem. Phys., 98, 4149 (1992).

2. R. Agrawal and D.A. Kofke, Phys. Rev. Lett., 74, 122 (1995).

3. R. Agrawal and D.A. Kofke, Mol. Phys., 85, 23 (1995).

4. R. Agrawal and D.A. Kofke, Mol. Phys., 85, 43 (1995).

5. E. J. Meijer and F. El Azhar, J. Chem. Phys., 106, 4678 (1996).

6. M. de Koning, A. Antonelli, and S. Yip, Phys. Rev. Lett., 83, 3973 (1999).

7. M. de Koning, A. Antonelli, and S. Yip, J. Comp. Aid. Mat. Design, 6, 349 (1999).

8. M. de Koning, W. Cai, A. Antonelli, and S. Yip, Comp. Sc. Eng., 2, 88 (2000).

9. D. Frenkel and B. Smit, Understanding Molecular Simulation From Algorithms to Applications, Academic Press, San Diego (1996).

10. M. de Koning and A. Antonelli, Phys. Rev. B, 55, 735 (1997).

11. J.E. Hunter III, W.P. Reinhardt, and T.F. Davis, J. Chem. Phys., 99, 6856 (1993) 